\title{
FULLY FUZZY PRIME SEMIGROUPS
}

M. SHABIR

Received 25 March 2004

We characterize those semigroups for which each fuzzy ideal is prime. We also characterize those semigroups for which each fuzzy right ideal is prime.

\section{Introduction and preliminaries}

The fundamental concept of a fuzzy set, introduced by Zadeh in his classic paper [5] of 1965 , has been applied by many authors to generalize some of the basic notions of algebra. In this note we characterize the semigroups for which each fuzzy ideal is prime, also the semigroups for which each fuzzy right ideal is prime.

In Section 2, we prove that a semigroup is fully fuzzy prime if and only if it is semisimple and its set of fuzzy ideals is totally ordered. In Section 3, we define the fuzzy prime right ideals of a semigroup and we prove that if the set of all fuzzy right ideals of $S$ is totally ordered, then $S$ is right weakly regular if and only if every fuzzy right ideal of $S$ is a fuzzy prime right ideal.

By a semigroup $(S, \cdot)$, we mean a nonempty set $S$ together with an associative binary operation ".". A semigroup $S$ is called commutative if "." is commutative, that is, $a \cdot b=$ $b \cdot a$ for all $a, b \in S$. A semigroup $S$ is called a monoid if it has an identity element with respect to ".". If $S$ has no identity element, then it is easy to adjoin an identity element 1 to the set by defining $1 \cdot s=s \cdot 1=s$, for all $s$ in $S$. We will use the notation $S^{1}$ with the following meaning:

$$
S^{1}= \begin{cases}S & \text { if } S \text { has an identity element, } \\ S \cup\{1\} & \text { otherwise. }\end{cases}
$$

A semigroup $S$ is called regular, if for each $a$ in $S$ there exists $x$ in $S$ such that $a x a=a$. If $a$ is an element of a semigroup $S$, we say that $x$ is an inverse of $a$ if $a x a=a$ and $x a x=x$. A semigroup $S$ is called an inverse semigroup if each of its elements has a unique inverse.

By a left (right) ideal of $S$, we mean a nonempty subset $A$ of $S$ such that $S A \subseteq A(A S \subseteq$ $A$ ). By a two-sided ideal or simply an ideal, we mean a nonempty subset of $S$, which is both a left and a right ideal of $S$. An ideal $P$ of a semigroup $S$ is called prime (semiprime) 
ideal of $S$ if for ideals $I, J$ of $S, I J \subseteq P\left(I^{2} \subseteq P\right)$ implies $I \subseteq P$ or $J \subseteq P(I \subseteq P) ; P$ is called irreducible if for ideals $I, J$ of $S, I \cap J=P$ implies $I=P$ or $J=P$.

A function $f$ from a nonempty set $A$ to the unit interval $[0,1]$ is called a fuzzy subset of $A$. A fuzzy subset $\lambda: A \rightarrow[0,1]$ is nonempty if $\lambda$ is not the constant map which assumes the value 0 . For fuzzy subsets $\lambda$ and $\mu$ of $A, \lambda \leq \mu$ means that for all $a \in A, \lambda(a) \leq \mu(a)$. The symbols $\lambda \wedge \mu$ and $\lambda \vee \mu$ denote the following fuzzy subsets of $A$ :

$$
\begin{array}{ll}
(\lambda \wedge \mu)(a)=\lambda(a) \wedge \mu(a) & (a \in A), \\
(\lambda \vee \mu)(a)=\lambda(a) \vee \mu(a) & (a \in A) .
\end{array}
$$

By the product $\lambda \circ \mu$ of two fuzzy subsets $\lambda, \mu$ of a semigroup $S$, we mean the following fuzzy subset:

$$
(\lambda \circ \mu)(x)= \begin{cases}\vee_{x=y z}(\lambda(y) \wedge \mu(z)) & \text { if } x \text { is expressible as } x=y z \\ 0 & \text { otherwise. }\end{cases}
$$

The operation $\circ$ is associative.

A nonempty fuzzy subset $f$ of $S$ is called a fuzzy left (right) ideal of $S$ if $f_{S} \circ f \leq f$, $\left(f \circ f_{S} \leq f\right)$, where $f_{S}: S \rightarrow[0,1]$ maps every element of $S$ to 1 . A nonempty fuzzy subset $f$ of $S$ is called a fuzzy two-sided ideal of $S$ if it is both a fuzzy left and a fuzzy right ideal of $S$.

The following lemmas are taken from [2].

LeMma 1.1. A fuzzy subset $f$ of a semigroup $S$ is a fuzzy left ideal of $S$ if and only if $f(x y) \geq$ $f(y)$ for all $x, y \in S$.

Lemma 1.2. Let $f$, $g$, and $h$ be fuzzy subsets of $S$, then

(i) $f \vee(g \wedge h)=(f \vee g) \wedge(f \vee h)$,

(ii) $f \wedge(g \vee h)=(f \wedge g) \vee(f \wedge h)$.

Lemma 1.3. Let $f, g$, and $h$ be fuzzy subsets of $S$, then

(i) $f \circ(g \vee h)=(f \circ g) \vee(f \circ h),(g \vee h) \circ f=(g \circ f) \vee(h \circ f)$,

(ii) $f \circ(g \wedge h) \leq(f \circ g) \wedge(f \circ h),(g \wedge h) \circ f \leq(g \circ f) \wedge(h \circ f)$.

Lemma 1.4. Let $f, g$, and $h$ be fuzzy subsets of S. If $f \leq g$ then $f \circ h \leq g \circ h$ and $h \circ f \leq$ $h \circ g$.

LEMMA 1.5. If $f$ is a nonempty fuzzy subset of $S$, then $f_{S} \circ f\left(f \circ f_{S}\right)$ is a fuzzy left (right) ideal of $S$.

LEMma 1.6. If $f$ is a fuzzy right (left) ideal of $S$, then $f \vee\left(f_{S} \circ f\right)\left(f \vee\left(f \circ f_{S}\right)\right)$ is a fuzzy two-sided ideal of $S$.

LEMmA 1.7. If $\lambda$ and $\mu$ are fuzzy ideals of $S$, then $\lambda \wedge \mu$ and $\lambda \vee \mu$ are fuzzy ideals of $S$.

Lemma 1.8. If $\lambda$ and $\mu$ are fuzzy ideals of $S$, then $\lambda \circ \mu$ is a fuzzy ideal of $S$ and $\lambda \circ \mu \leq \lambda \wedge \mu$. 


\section{Fully fuzzy prime semigroups}

Definition 2.1. A semigroup $S$ is called semisimple if each of its ideals is idempotent.

Definition 2.2. A fuzzy ideal $\xi$ of a semigroup $S$ is called fuzzy prime ideal of $S$ if for any fuzzy ideals $\lambda$, $\mu$ of $S, \lambda \circ \mu \leq \xi$ implies $\lambda \leq \xi$ or $\mu \leq \xi$, $\xi$ is called fuzzy irreducible if for any fuzzy ideals $\lambda$, $\mu$ of $S, \lambda \wedge \mu=\xi$ implies $\lambda=\xi$ or $\mu=\xi$. A fuzzy ideal $\xi$ of a semigroup $S$ is called fuzzy semiprime idealof $S$ if for any fuzzy ideal $\lambda$ of $S, \lambda^{2} \leq \xi$ implies $\lambda \leq \xi$.

Proposition 2.3. A fuzzy ideal of a semigroup $S$ is fuzzy prime ideal if and only if it is fuzzy semiprime and fuzzy irreducible.

Proof. Let $\lambda$ be a fuzzy prime ideal of a semigroup $S$, then $\lambda$ is fuzzy semiprime ideal. Let $\mu, \nu$ be fuzzy ideals of $S$ such that $\mu \wedge \nu=\lambda$. Since $\mu \circ \nu \leq \mu \wedge \nu=\lambda$ and $\lambda$ is fuzzy prime ideal of $S$, we have $\mu \leq \lambda$ or $\nu \leq \lambda$. Since $\lambda \leq \mu$ and $\lambda \leq \nu$, we have $\lambda=\mu$ or $\lambda=\nu$. Hence $\lambda$ is irreducible.

Conversely, assume that $\lambda$ is a fuzzy ideal of $S$, which is both fuzzy semiprime and fuzzy irreducible. If $\mu, v$ are fuzzy ideals of $S$ such that $\mu \circ \nu \leq \lambda$, then $(\mu \wedge \nu)^{2} \leq \mu \circ \nu \leq \lambda$. Since $\lambda$ is fuzzy semiprime, we have $\mu \wedge \nu \leq \lambda$. Thus $(\mu \wedge \nu) \vee \lambda=\lambda$. This implies that $(\mu \vee \lambda) \wedge(\nu \vee \lambda)=\lambda$. Since $\lambda$ is fuzzy irreducible, we have $\mu \vee \lambda=\lambda$ or $\nu \vee \lambda=\lambda$. That is either $\mu \leq \lambda$, or $\nu \leq \lambda$.

Definition 2.4. A fuzzy ideal $\lambda$ of a semigroup $S$ is called idempotent if $\lambda \circ \lambda=\lambda$.

Definition 2.5. A semigroup $S$ is called fully fuzzy prime (semiprime) if each of its fuzzy ideal is prime (semiprime).

THEOREM 2.6. The following assertions on a semigroup $S$ are equivalent:

(1) $S$ is semisimple,

(2) each fuzzy ideal of $S$ is idempotent,

(3) for each pair of fuzzy ideals $\lambda$, $\mu$ of $S, \lambda \wedge \mu=\lambda \circ \mu$,

(4) the set of all fuzzy ideals of $S$ (ordered by inclusion) is a distributive lattice under the union and product of fuzzy ideals,

(5) each proper fuzzy ideal of $S$ is the intersection of fuzzy prime ideals,

(6) each fuzzy ideal of $S$ is fuzzy semiprime ideal.

Proof. (1) $\Leftrightarrow(2) \Leftrightarrow(3)$. This is due to Kuroki [4].

$(1) \Leftrightarrow(4) \Leftrightarrow(5)$. This is proved in [3].

$(1) \Rightarrow(6)$. Let $\lambda$ be a fuzzy ideal of $S$. Let $\delta$ be a fuzzy ideal of $S$ such that $\delta^{2} \leq \lambda$. Now by (1), $\delta^{2}=\delta$, so $\delta \leq \lambda$. Hence, $\lambda$ is fuzzy semiprime ideal.

$(6) \Rightarrow(1)$. Let $\lambda$ be any fuzzy ideal of $S$. Then $\lambda^{2}$ is also a fuzzy ideal of $S$. Since $\lambda^{2} \leq \lambda^{2}$, by (6), $\lambda \leq \lambda^{2}$. We have on the other hand $\lambda^{2} \leq \lambda$, so $\lambda=\lambda^{2}$.

Theorem 2.7. Let $S$ be a semisimple semigroup. For a fuzzy ideal $\xi$ of $S$, the following conditions are equivalent:

(1) $\xi$ is a fuzzy prime ideal,

(2) $\xi$ is a fuzzy irreducible ideal. 
Proof. Assume that $\xi$ is a fuzzy prime ideal of $S$. We show that $\xi$ is fuzzy irreducible, that is, for fuzzy ideals $\lambda, \mu$ of $S, \lambda \wedge \mu=\xi$ implies $\lambda=\xi$ or $\mu=\xi$. Since $\lambda \wedge \mu=\xi$, it follows that $\xi \leq \lambda$ and $\xi \leq \mu$. By Theorem 2.6, $\lambda \circ \mu=\lambda \wedge \mu$. Hence, $\xi=\lambda \wedge \mu=\lambda \circ \mu$. Since $\xi$ is a fuzzy prime ideal, we have either $\lambda \leq \xi$ or $\mu \leq \xi$. So $\xi=\lambda$ or $\xi=\mu$.

Conversely, assume that $\xi$ is a fuzzy irreducible ideal. We show that $\xi$ is a fuzzy prime ideal. Suppose that there exist fuzzy ideals $\lambda$ and $\mu$ such that $\lambda \circ \mu \leq \xi$. By Theorem 2.6, $\lambda \circ$ $\mu=\lambda \wedge \mu$. Thus, $(\lambda \wedge \mu) \vee \xi=\xi$ implies $(\lambda \vee \xi) \wedge(\mu \vee \xi)=\xi$. Since $\xi$ is fuzzy irreducible, it follows that either $\lambda \vee \xi=\xi$ or $\mu \vee \xi=\xi$. Then we have $\lambda \leq \xi$ or $\mu \leq \xi$. Hence, $\xi$ is a fuzzy prime ideal.

THeORem 2.8. A semigroup $S$ is fully fuzzy prime if and only if $S$ is semisimple and the set of fuzzy ideals of $S$ is totally ordered.

Proof. Suppose $S$ is a fully fuzzy prime semigroup, $\lambda$ is a fuzzy ideal of $S$, then $\lambda^{2}$ is also a fuzzy ideal of $S$. Since $\lambda^{2} \leq \lambda^{2}$, we have $\lambda \leq \lambda^{2}$. On the other hand, $\lambda^{2} \leq \lambda$. Hence $\lambda^{2}=\lambda$. Thus, every fuzzy ideal of $S$ is idempotent, so $S$ is semisimple. Let $\lambda$ and $\mu$ be fuzzy ideals of $S$, then $\lambda \wedge \mu$ is a fuzzy ideal and so fuzzy prime ideal of $S$. Since $\lambda \circ \mu \leq \lambda \wedge \mu$, then either $\lambda \leq \lambda \wedge \mu$ or $\mu \leq \lambda \wedge \mu$. Hence, $\lambda \leq \mu$ or $\mu \leq \lambda$.

Conversely, assume that $S$ is a semisimple semigroup and the set of fuzzy ideals of $S$ is totally ordered. Let $\lambda, \mu$, and $\xi$ be fuzzy ideals of $S$ such that $\lambda \circ \mu \leq \xi$. Since the set of fuzzy ideals of $S$ is totally ordered, so we have $\lambda \leq \mu$ or $\mu \leq \lambda$. Assume that $\lambda \leq \mu$. Now $\lambda=\lambda^{2} \leq \lambda \circ \mu \leq \xi$. Thus, $\xi$ is a fuzzy prime ideal of $S$.

Corollary 2.9. A commutative semigroup $S$ is fully fuzzy prime if and only if $S$ is an inverse semigroup and the set of fuzzy ideals of $S$ is totally ordered.

Proof. Since each commutative semisimple semigroup is an inverse semigroup, the corollary follows from the above theorem.

\section{Fuzzy prime right ideals}

Throughout this section, $S$ will denote a monoid, that is, a semigroup with an identity 1 , which also contains a two-sided zero.

Definition 3.1. A fuzzy right ideal $\xi$ of a semigroup $S$ is called fuzzy prime right ideal of $S$ if for any fuzzy right ideals $\lambda, \mu$ of $S, \lambda \circ \mu \leq \xi$ implies $\lambda \leq \xi$ or $\mu \leq \xi$; $\xi$ is called fuzzy irreducible if for any fuzzy right ideals $\lambda, \mu$ of $S, \lambda \wedge \mu=\xi$ implies $\lambda=\xi$ or $\mu=\xi$. A fuzzy right ideal $\xi$ of a semigroup $S$ is called fuzzy semiprime right ideal of $S$ if for any fuzzy right ideal $\lambda$ of $S, \lambda^{2} \leq \xi$ implies $\lambda \leq \xi$.

Definition 3.2. A semigroup $S$ is called right weakly regular if for each $x \in S, x \in(x S)^{2}$ (cf. [1]).

LEMMA 3.3. If $S$ is a semigroup, then the intersection of fuzzy prime right ideals of $S$ is a fuzzy semiprime right ideal.

Lemma 3.4. Let $S$ be a semigroup. A fuzzy semiprime irreducible right ideal of $S$ is a fuzzy prime right ideal. 
Proof. Let $\xi$ be a fuzzy semiprime irreducible right ideal of $S$. Let $\lambda$, $\mu$ be fuzzy right ideals of $S$, such that $\lambda \circ \mu \leq \xi$. Now we show that $\mu \leq f_{S} \circ \mu$. Let $x \in S$. Then $\left(f_{S} \circ \mu\right)(x)=$ $\vee_{x=y z}\left\{f_{S}(y) \wedge \mu(z)\right\}=\vee_{x=y z}\{\mu(z)\}$ because $f_{S}(y)=1 \geq \mu(x)$, as we have $x=1 x$.

Hence, $\mu \vee f_{S} \circ \mu=f_{S} \circ \mu$ is a fuzzy ideal of $S$. Also $\lambda \wedge f_{S} \circ \mu$ is a fuzzy right ideal of $S$.

Now $\left(\lambda \wedge f_{S} \circ \mu\right)^{2} \leq \lambda \circ\left(f_{S} \circ \mu\right)=\left(\lambda \circ f_{S}\right) \circ \mu \leq \lambda \circ \mu \leq \xi$.

Since $\xi$ is fuzzy semiprime right ideal, $\lambda \wedge f_{S} \circ \mu \leq \xi$. Thus $\left(\lambda \wedge f_{S} \circ \mu\right) \vee \xi=\xi$ implies $(\lambda \vee \xi) \wedge\left(f_{S} \circ \mu \vee \xi\right)=\xi$.

Since $\xi$ is fuzzy irreducible, we have $(\lambda \vee \xi)=\xi$ or $\left(f_{S} \circ \mu \vee \xi\right)=\xi$ that is either $\lambda \leq \xi$ or $f_{S} \circ \mu \leq \xi$ implies either $\lambda \leq \xi$ or $\mu \leq \xi$. Hence, $\xi$ is a fuzzy prime right ideal of $S$.

THEOREM 3.5. The following assertions on a semigroup $S$ are equivalent.

(1) $S$ is right weakly regular,

(2) each fuzzy right ideal of $S$ is idempotent,

(3) for each fuzzy ideal $\mu$ and for each fuzzy right ideal $\lambda$ of $S, \lambda \wedge \mu=\lambda \circ \mu$,

(4) each fuzzy right ideal of $S$ is a fuzzy semiprime right ideal,

(5) each fuzzy right ideal is the intersection of those fuzzy prime right ideals of $S$ which contain it.

Proof. $(1) \Leftrightarrow(2) \Leftrightarrow(3)$. Compare to [1].

$(1) \Rightarrow(4)$. Let $\xi$ be a fuzzy right ideal of $S$. Let $\lambda^{2} \leq \xi$, where $\lambda$ is a fuzzy right ideal of $S$. By (2) $\lambda^{2}=\lambda$, so $\lambda \leq \xi$. Thus $\xi$ is a fuzzy semiprime right ideal of $S$.

$(4) \Rightarrow(5)$. First, we show that each fuzzy right ideal of $S$ is contained in a fuzzy irreducible right ideal. Let $\lambda$ be a fuzzy right ideal of $S$ with $\lambda(a)=\alpha$, where $a$ is any element of $S$ and $\alpha \in[0,1]$. Let $X=\{\mu: \mu$ is a fuzzy right ideal of $S, \mu(a)=\alpha$, and $\lambda \leq \mu\}$. Then $X \neq \varnothing$ since $\lambda \in X$. Define $\xi=\vee X$, then $\xi$ is a maximal fuzzy right ideal of $S$ satisfying $\xi(a)=\alpha$. We now show that $\xi$ is a fuzzy irreducible right ideal of $S$. Suppose $\xi=\delta_{1} \wedge \delta_{2}$, where $\delta_{1}$ and $\delta_{2}$ are fuzzy right ideals of $S$. This implies that $\xi \leq \delta_{1}$ and $\xi \leq \delta_{2}$. We claim that either $\xi=\delta_{1}$ or $\xi=\delta_{2}$. Suppose, on the contrary, $\xi \neq \delta_{1}$ and $\xi \neq \delta_{2}$. Since $\xi$ is maximal with respect to the property that $\xi(a)=\alpha$ and since $\xi \leqq \delta_{1}$ and $\xi \varsubsetneqq \delta_{2}$, it follows that $\delta_{1}(a) \neq \alpha$ and $\delta_{2}(a) \neq \alpha$. Hence $\alpha=\xi(a)=\left(\delta_{1} \wedge \delta_{2}\right)(a)=\left\{\delta_{1}(a) \wedge \delta_{2}(a)\right\} \neq \alpha$, which is impossible. Hence, $\xi=\delta_{1}$ or $\xi=\delta_{2}$. This proves that $\xi$ is a fuzzy irreducible ideal.

Now let $\lambda$ be a fuzzy right ideal of $S$ and let $\left\{\lambda_{\alpha}: \alpha \in \Omega\right\}$ be the family of fuzzy irreducible right ideals of $S$ which contain $\lambda$. Obviously, $\lambda \leq \wedge_{\alpha \in \Omega} \lambda_{\alpha}$. We now prove that $\wedge_{\alpha \in \Omega} \lambda_{\alpha} \leq \lambda$. Let $a$ be any element of $S$. Then there exists a fuzzy irreducible right ideal, say $\lambda_{\beta}$, such that $\lambda \leq \lambda_{\beta}$ and $\lambda(a)=\lambda_{\beta}(a)$. Thus $\lambda_{\beta} \in\left\{\lambda_{\alpha}: \alpha \in \Omega\right\}$. Hence $\wedge_{\alpha \in \Omega} \lambda_{\alpha} \leq \lambda_{\beta}$, so $\wedge_{\alpha \in \Omega} \lambda_{\alpha}(a) \leq \lambda_{\beta}(a)=\lambda(a)$. This implies that $\wedge_{\alpha \in \Omega} \lambda_{\alpha} \leq \lambda$. Hence, each fuzzy right ideal of $S$ can be written as the intersection of fuzzy irreducible right ideals of $S$ which contain it. By (4), each fuzzy right ideal is semiprime, so by Lemma 3.4, each fuzzy right ideal of $S$ is the intersection of fuzzy prime right ideals of $S$ which contain it.

$(5) \Rightarrow(1)$. Let $\lambda$ be a fuzzy right ideal of $S$, then $\lambda^{2}$ is also a fuzzy right ideal of $S$. By hypothesis, $\lambda^{2}$ is an intersection of fuzzy prime right ideals and so is a fuzzy semiprime right ideal of $S$. As $\lambda^{2} \leq \lambda^{2}$, so $\lambda \leq \lambda^{2}$. On the other hand, $\lambda^{2} \leq \lambda$. Hence $\lambda^{2}=\lambda$. As (2) is equivalent to (1), thus $S$ is right weakly regular.

THeOREM 3.6. If every fuzzy right ideal of $S$ is fuzzy prime right ideal, then $S$ is right weakly regular and the set of fuzzy ideals of $S$ is totally ordered. 
Proof. Suppose $S$ is a semigroup in which each fuzzy right ideal is prime and let $\lambda$ be a fuzzy right ideal of $S$. Then $\lambda^{2}$ is also a fuzzy right ideal of $S$. Since $\lambda^{2} \leq \lambda^{2}$, we have $\lambda \leq \lambda^{2}$. On the other hand $\lambda^{2} \leq \lambda$. Hence $\lambda^{2}=\lambda$. Thus, every fuzzy right ideal of $S$ is idempotent, so $S$ is right weakly regular. Let $\lambda$ and $\mu$ be any fuzzy ideals of $S$. Since $\lambda \circ \mu \leq \lambda \wedge \mu$. As $\lambda \wedge \mu$ is a fuzzy ideal of $S$, then it is, a fuzzy prime ideal. Thus either $\lambda \leq \lambda \wedge \mu$ or $\mu \leq \lambda \wedge \mu$, and therefore either $\lambda \leq \mu$ or $\mu \leq \lambda$.

PROPOSITION 3.7. If $S$ is a right weakly regular semigroup and the set of all fuzzy right ideals of $S$ is totally ordered, then every fuzzy right ideal of $S$ is a fuzzy prime right ideal.

Proof. Let $\lambda, \mu$, and $\xi$ be fuzzy right ideals of $S$ such that $\lambda \circ \mu \leq \xi$. Since the set of all fuzzy right ideals of $S$ is totally ordered, we have $\lambda \leq \mu$ or $\mu \leq \lambda$. If $\lambda \leq \mu$, then $\lambda=\lambda^{2} \leq \lambda \circ \mu \leq \xi$. If $\mu \leq \lambda$, then $\mu=\mu^{2} \leq \lambda \circ \mu \leq \xi$. Thus $\xi$ is a fuzzy prime right ideal.

THeOREm 3.8. If the set of fuzzy right ideals of $S$ is totally ordered, then the following are equivalent.

(1) $S$ is right weakly regular,

(2) every fuzzy right ideal of $S$ is a fuzzy prime right ideal.

\section{References}

[1] J. Ahsan, M. F. Khan, and M. Shabir, Characterizations of monoids by the properties of their fuzzy subsystems, Fuzzy Sets and Systems 56 (1993), no. 2, 199-208.

[2] J. Ahsan, R. M. Latif, and M. Shabir, Fuzzy quasi-ideals in semigroups, J. Fuzzy Math. 9 (2001), no. 2, 259-270.

[3] J. Ahsan, K. Saifullah, and M. F. Khan, Semigroups characterized by their fuzzy ideals, Mohu Xitong yu Shuxue 9 (1995), no. 1, 29-32.

[4] N. Kuroki, On fuzzy semigroups, Inform. Sci. 53 (1991), no. 3, 203-236.

[5] L. A. Zadeh, Fuzzy sets, Inform. and Control 8 (1965), no. 3, 338-353.

M. Shabir: Department of Mathematics, Quaid-i-Azam University, Islamabad-45320, Pakistan E-mail address: msabirbhatti@yahoo.co.uk 


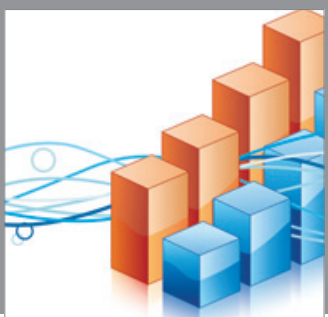

Advances in

Operations Research

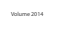

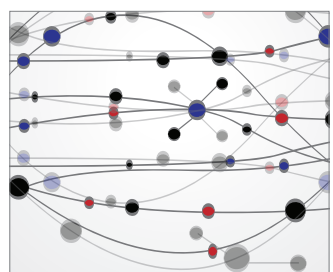

\section{The Scientific} World Journal
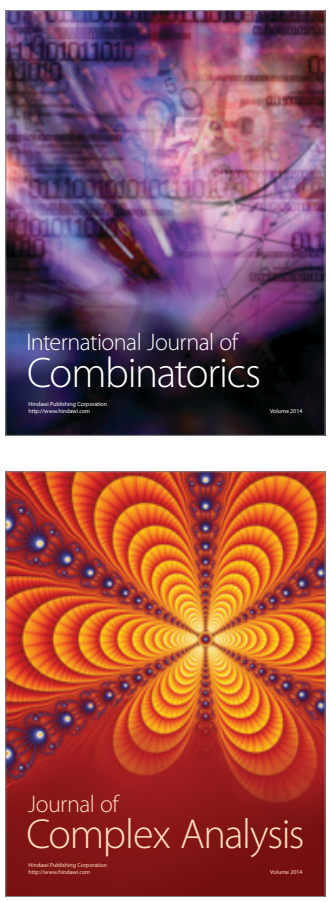

International Journal of

Mathematics and

Mathematical

Sciences
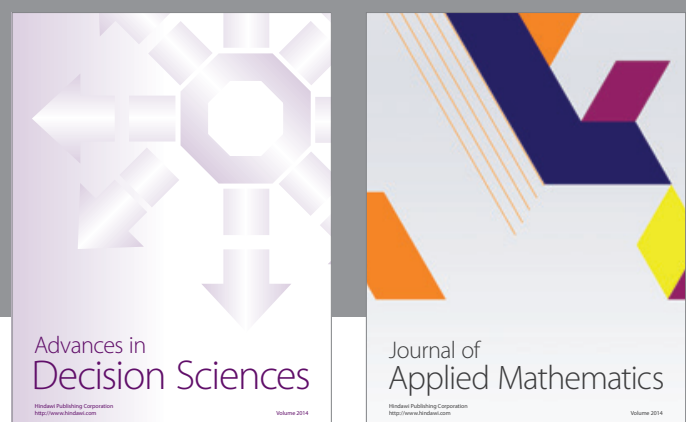

Journal of

Applied Mathematics
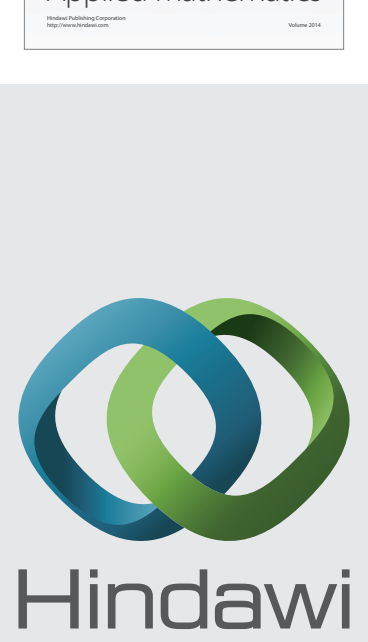

Submit your manuscripts at http://www.hindawi.com
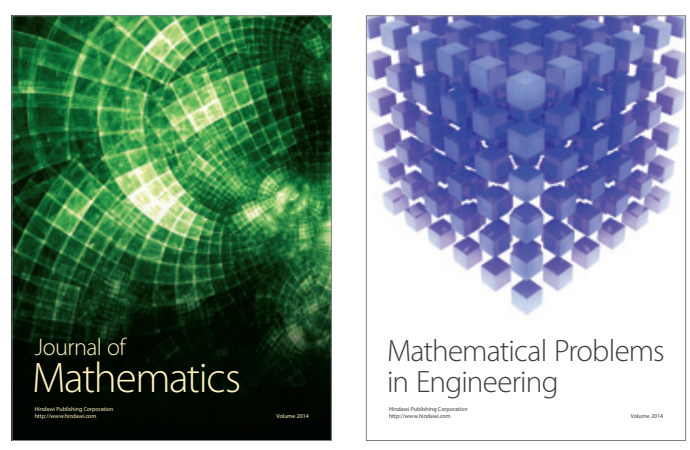

Mathematical Problems in Engineering
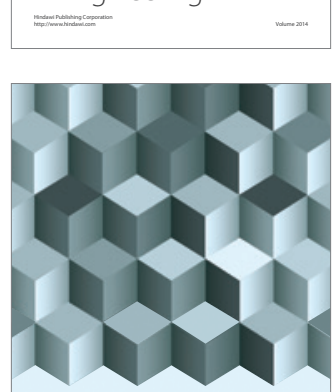

Journal of

Function Spaces
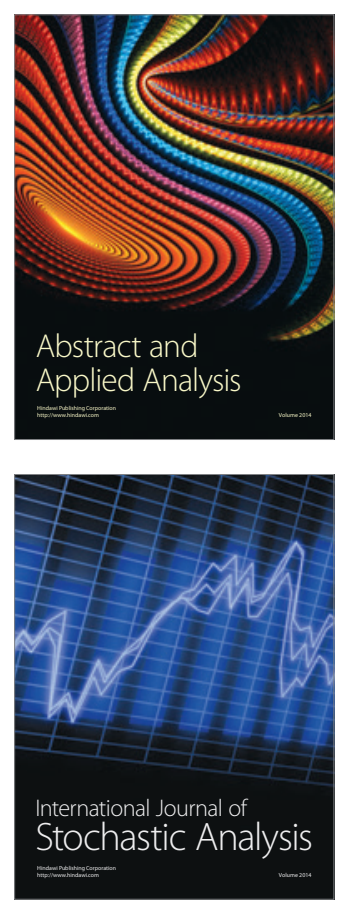

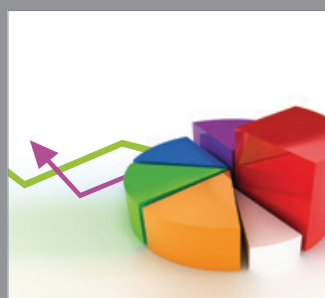

ournal of

Probability and Statistics

Promensencen
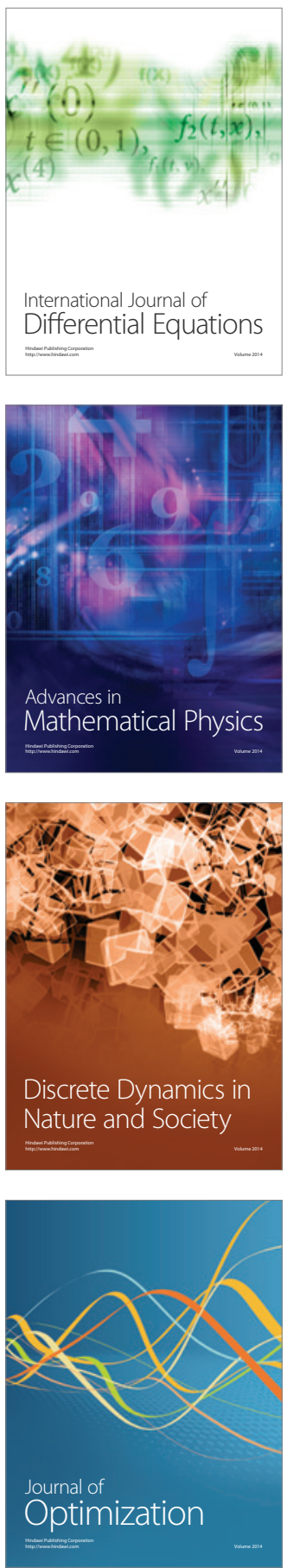